\section{Micromanaging oncolytic viruses}

\section{By Tim Fulmer, Senior Writer}

Oncolytic viruses have had limited success as cancer therapies, largely because of the need to balance potent antitumor activity with off-target toxicity. Two research teams have now applied a microRNA-based strategy that could restrict the replication of oncolytic viruses to tumors without the need for alterations to the viral genome that can lead to an overly attenuated strain. ${ }^{1,2}$

Oncolytic viruses are engineered to selectively target and replicate within tumor cells, triggering lysis and cell death. Selective targeting is usually achieved by mutating the viral genome to lower the likelihood that the virus will target or replicate within healthy cells. However, these mutations carry the risk of weakening the virus strain's ability to replicate within the tumor. Thus, attempts to balance potency and off-target pathogenicity have led to oncolytic viruses that are safe but lack efficacy in the clinic (see

Table 1, "Oncolytic virus therapies").,3

Enter miRNAs, which are single-stranded, noncoding RNA molecules that downregulate gene expression by targeting specific mRNAs and preventing protein translation.

The key is that miRNA expression patterns are often tissue-specific. Thus, the two groups of researchers separately hypothesized that an oncolytic virus encoding sequences targeted by a tissue-specific miRNA would be prevented from replicating specifically in that tissue. This could be achieved by inserting the miRNA target sequence adjacent to genes required for viral replication.

The hope was that the virus would have limited off-target pathogenicity without the need for genetic attenuation.

In a paper in Nature Medicine, Stephen Russell and colleagues at the Mayo Clinic College of Medicine focused on coxsackievirus A21 (CVA21). The single-stranded RNA virus has previously shown activity in mouse models of cancer but has also caused fatal paralysis via inflammation and necrosis of skeletal muscle.

To address this toxicity, the researchers created a recombinant CVA21 strain that expressed sequences targeted by two muscle-specific miRNAs, miRNA-133 and miRNA-206.

In mouse models of multiple myeloma (MM), a single intratumoral dose of the recombinant CVA21 strain produced a decrease in tumor volume comparable to that seen using wild-type virus. Moreover, mice receiving the recombinant CVA21 strain had less muscle necrosis and significantly greater survival $(p<0.001)$ than littermates that received wild-type CVA21, all of which developed hind-limb paralysis and required killing.

The authors concluded that incorporation of tissue-specific miRNA targets into oncolytic viruses "might increase their therapeutic index without perturbing their antitumor activity."

While the Mayo researchers focused on limiting muscle toxicity, a paper in the Journal of Virology describes a strategy to eliminate liver toxicity associated with systemic delivery of oncolytic adenoviruses.

In that paper, researchers at the University of Helsinki and the University of California, San Francisco, constructed a recombinant adenovirus that contained a target sequence for liver-specific miRNA-122.

For a model, the researchers chose a liver cell line that expresses high levels of miRNA-122. Infection of those cells with a recombinant adenoviral strain containing three copies of the miRNA-122 target sequence inserted at a site adjacent to genes required for viral replication led to undetectable levels of adenoviral proteins.

"The papers potentially usher in a new era of oncolytic virus regulation," said John Bell, CSO of oncolytic virus company Jennerex Biotherapeutics Inc. "This approach may allow scientists to design viruses that maintain potency without having off-target infections." Bell is also a senior scientist at the Ottawa Health Research Institute and a professor of medicine at the University of Ottawa.

Jennerex's JX-594, a recombinant vaccinia virus (thymidine kinase deletion plus immunostimulatory granulocyte/macrophage colonystimulating factor (GM-CSF)), is in a Phase II trial to treat unresectable hepatocellular carcinoma (HCC) and a Phase I trial to treat refractory solid tumors.

\section{Safe and stable}

Despite the potential for fewer side effects, Jennerex CEO David Kirn said he was concerned that the miRNA targeting sequences are genetically unstable and could potentially mutate. That would cause the strain to revert back to a form that is toxic to normal tissue.

Bell agreed that escape mutants were a concern but added that "incorporation of multiple miRNAs throughout the viral genome might help mitigate the problem, and even when rare reversions arise, they could be eliminated by innate or adaptive immune responses."

Indeed, the Nature Medicine authors reported that CVA21 strains isolated from 6 of 11 infected mice were genetically identical to the original recombinant strain 45 days after virus administration. Virus isolated from three of the five remaining mice had at least $80 \%$ homology in the miRNA targeting regions compared with the same regions in the original recombinant strain.

Of the mice that had a mutated miRNA insert, 2 were free of disease symptoms at the end of the 70-day study. Only 1 of the 11 isolated strains had significantly lower retention of the miRNA targeting sequence. 
Table 1. Oncolytic virus therapies. At least 13 oncolytic virus therapies are in development for various cancers.

\begin{tabular}{|c|c|c|c|c|}
\hline Company & Product & Description & Indication & Status \\
\hline Shanghai Sunway Biotech Co. Ltd. & Oncorine & $\begin{array}{l}\text { Modified adenovirus with deletion of an } \\
\text { E1B-55kd segment }\end{array}$ & Head and neck cancer & $\begin{array}{l}\text { Marketed } \\
\text { (China) }\end{array}$ \\
\hline BioVex Inc. & $\begin{array}{l}\text { OncoVEX } \\
\text { GM-CSF }\end{array}$ & $\begin{array}{l}\text { Modified herpes simplex virus (HSV) } \\
\text { encoding granulocyte/macrophage colony- } \\
\text { stimulating factor (GM-CSF) }\end{array}$ & Various cancers & Phase II \\
\hline Jennerex Biotherapeutics Inc. & JX-594 & $\begin{array}{l}\text { Recombinant vaccinia virus (thymidine } \\
\text { kinase deletion plus GM-CSF) }\end{array}$ & Various cancers & Phase II \\
\hline MediGene AG (Xetra:MDG) ${ }^{\mathrm{A}}$ & NV1020 & Engineered HSV & Liver metastasis & Phase II \\
\hline $\begin{array}{l}\text { Oncolytics Biotech Inc. (TSX:ONC; } \\
\text { NASDAQ:ONCY) }\end{array}$ & Reolysin & Formulation of human reovirus type 3 & Various cancers & Phase II \\
\hline Jennerex & JX-929 & $\begin{array}{l}\text { Recombinant vaccinia virus carrying } \\
\text { cytosine deaminase }\end{array}$ & Cancer & Phase I \\
\hline MediGene $^{A}$ & G207 & $\begin{array}{l}\text { HSV modified to produce a toxic metabolite } \\
\text { in tumor cells }\end{array}$ & Brain cancer & Phase I \\
\hline Neotropix Inc. & NTX-010 & Naturally occurring oncolytic picornavirus & Cancer & Phase I \\
\hline Viralytics Ltd. (ASX:VLA) & Cavatek & Coxsackievirus A21 (CVA21) & Melanoma; solid tumors & Phase I \\
\hline M’s Science Corp. & HF10 & Mutant of HSV-1 & Head and neck cancer & Pilot (Japan) \\
\hline Genelux Corp. & GL-ONC1 & $\begin{array}{l}\text { Genetically stable modified vaccinia } \\
\text { oncolytic virus }\end{array}$ & Breast cancer & Preclinical \\
\hline $\begin{array}{l}\text { Introgen Therapeutics Inc. } \\
\text { (NASDAQ:INGN) }\end{array}$ & INGN 007 & $\begin{array}{l}\text { Replicating adenovirus vector overexpressing } \\
\text { adenosine diphosphate }\end{array}$ & Cancer & Preclinical \\
\hline Jennerex & JX-963 & Recombinant vaccinia virus & Cancer & Preclinical \\
\hline
\end{tabular}

Kalle Saksela, corresponding author on the Journal of Virology paper, told SciBX that his lab "observed no adenoviral escape mutants during the two-week in vitro infection experiments, suggesting that the reversion rate is rather slow and that the number of reversion mutants, when they arise, is probably small enough to be cleared by the immune system without serious side effects."

Saksela is chairman of the Department of Virology at the University of Helsinki.

Paul Hallenbeck, president and CEO of Neotropix Inc., said another potential challenge facing the miRNA-based strategy is lowering toxicity in multiple types of healthy tissues.

"For instance, in the Journal of Virology paper, the strategy resulted only in reducing viral replication in liver cells. However, while the liver is indeed a major organ of toxicity, the strategy is likely not to have changed the fact that adenoviruses replicate and cause toxicity in many other normal tissues," Hollenbeck said.

Saksela responded that "our main goal was to prevent the acute hepatotoxicity that has been associated with the systemic delivery of oncolytic adenoviral strains and adenoviral vectors in primates and humans. The risk of toxicity to other tissues will clearly be a function of the levels of virus that are administered. At the levels we are delivering, the main concern is clearly liver toxicity."

Neotropix's NTX-010, a tumor-targeting single-stranded RNA virus, is in a Phase I trial in patients with small cell lung cancer (SCLC). Early next year, the company hopes to begin a Phase II SCLC trial and a Phase
I/II trial in neuroblastoma and other solid pediatric cancers.

As to the Nature Medicine findings, Hallenbeck similarly thinks the modified CVA21 strain may have to address safety issues that extend beyond the muscles. "CVA21 not only causes muscle toxicity but also causes respiratory infections that may not be altered by the strategy in the Nature Medicine paper," he said.

In the only reported instance of CVA21 in patients-a Phase I safety trial of intratumoral wild-type CVA21 to treat late stage melanoma-
"The beauty of the miRNA strategy is that it can be combined with a variety of tumor-targeting strategies and is potentially applicable to any RNA or DNA virus."

-Kalle Saksela,

University of Helsinki Australian biotech Viralytics Ltd. did not report any serious off-target tissue toxicities.

Aladar Szalay, president and CEO of Genelux Corp., said he wanted to see more efficacy data. In particular, he's looking for data on the host immune response to miRNA-based oncolytic viruses.

"A key aspect of any oncolytic virus strategy is its ability to trigger an acute inflammation response at the site of the tumor," he said.

"Indeed, oncolysis in combination with a host innate immune response is generally much more effective than oncolysis alone for tumor regression and shrinkage."

Thus, said Szalay, who is also professor of medicine at University of Würzburg, "I would like to see proof that the miRNA-modified Coxsackievirus strain in the Nature Medicine paper replicates sufficiently within tumors to cause release of tumor antigens and tumor debris into the microenvironment and the subsequent triggering of a robust immune response."

Genelux’s GL-ONC1 (GLV-1h68), a genetically attenuated vaccinia 
virus that induces oncolysis and also expresses a luciferase-GFP fusion protein for noninvasive tumor imaging, is in preclinical testing. The strain has shown activity in murine xenograft breast cancer models following i.v. delivery. ${ }^{5}$

\section{Next steps}

Saksela said he is interested in combining the miRNA with other tumortargeting approaches to create an optimal oncolytic adenovirus.

"For example, the liver-specific miRNA silencing sequence could be combined with additional mutational modifications that limit adenoviral entry and/or replication to the tumor," he said. "The beauty of the miRNA strategy is that it can be combined with a variety of tumortargeting strategies and is potentially applicable to any RNA or DNA virus," he said.

Saksela said his group has used the same miRNA-based approach to restrict poliovirus from replicating in the mouse $\mathrm{CNS}$, thereby producing an attenuated strain that could potentially be used in a vaccine. ${ }^{6}$

Additional next steps for Saksela and colleagues include better characterization of miRNA expression in different tissues.

"For the most part, the published literature on tissue-specific miRNAs is rich enough to provide potential candidate sequences for miRNA targeting. However, tissue specificity by itself may not be enough to ensure the strategy's success. We suspect that the expression level of a given miRNA in its tissue will also impact the effectiveness of the approach," he said.

Over the longer term, said Saksela, researchers eventually may identify miRNAs that are downregulated or entirely absent in certain tumors but expressed in most normal cells. "An oncolytic virus targeted by such an miRNA would be prevented from replicating in all cells except the tumor and thus might represent the ideal tumor-targeted therapy," he said.

Indeed, Bell and colleagues at the University of Ottawa are doing just that: his group is taking advantage of let-7 miRNA, which is abundantly expressed in most mammalian cells but downregulated in some cancers.
They found that an oncolytic vesicular stomatitis virus (VSV) expressing let-7 targeting sequences had lower replication in normal cells while retaining oncolytic activity in cell culture and murine cancer models. ${ }^{7}$

Stephen Russell, corresponding author on the Nature Medicine paper and professor of medicine at the Mayo Clinic, told SciBX a patent application has been filed for the miRNA targeting strategy and is available for licensing. He also said he hopes to secure funding to begin testing the strategy in the clinic.

The Journal of Virology findings are not patented, according to Saksela.

Fulmer, T. SciBX 1(42); doi:10.1038/scibx.2008.1014

Published online Nov. 20, 2008

\section{REFERENCES}

1. Kelly, E. et al. Nat. Med.; published online Oct. 26, 2008; doi:10.1038/nm.1776

Contact: Stephen Russell, Mayo Clinic College of Medicine, Rochester, Minn. e-mail: sjr@mayo.edu

2. Ylosmaki, E. et al. J. Virol.; published online Sept. 17, 2008; doi:10.1128/JVI.01608-08

Contact: Kalle Saksela, University of Helsinki, Helsinki, Finland e-mail: kalle.saksela@helsinki.fi

3. Parato, K. et al. Nat. Rev. Cancer 5, 965-976 (2005)

4. Liu, T. \& Kirn, D. Gene Therapy 15, 877-884 (2008)

5. Zhang, Q. et al. Cancer Res. 67, 10038-10046 (2007)

6. Barnes, D. et al. Cell Host Microbe 4, 239-248 (2008)

7. Edge, R. et al. Mol. Ther. 16, 1437-1443 (2008)

COMPANIES AND INSTITUTIONS MENTIONED

Genelux Corp., San Diego, Calif.

Jennerex Biotherapeutics Inc., San Francisco, Calif. Mayo Clinic College of Medicine, Rochester, Minn. Neotropix Inc., Malvern, Pa.

Ottawa Health Research Institute, Ottawa, Ontario, Canada University of California, San Francisco, San Francisco, Calif. University of Helsinki, Helsinki, Finland

University of Ottawa, Ottawa, Ontario, Canada

University of Würzburg, Würzburg, Germany

Viralytics Ltd., Pymble, New South Wales, Australia 